\title{
Polymorphic Subtype Inference: Closing the Theory-Practice Gap
}

\author{
You-Chin Fuh Prateek Mishra \\ Department of Computer Science \\ The State University of New York at Stony Brook \\ Stony Brook, New York 11794-4400 \\ ARPANET: yfuh@suny.sbcs.edu, mishra@suny.sbcs.edu
}

\section{Introduction}

The theory of polymorphic type inference[Mil78] has been extended to deal with subtypes: Mitchell gave a subtype extension [Mit84], and we have given algorithms and correctness proofs for several key components of a type inference system based on Mitchell's extension [FM88]. However, the actual implementation of a subtype inference system diverges from these theoretical underpinnings in fundamentally important ways. By examining this divergence, we close the gap between the theoretical foundations of subtype inference and its actual use.

We give two results: first, we observe that Mitchell's instance relation is exactly a preorder and hence principal types are unique only up to equivalence. We show the existence of a unique minimal representative for each equivalence class of typings and give an algorithm for computing such representatives. In practice, we find it unnecessary to transform typings into their exact minimal forms. Instead, it is sufficient to remove certain redundancies from typings; this transforms most typings into their exact minimal form. We describe an efficient algorithm that implements this transformation and prove its correctness.

Second, we propose a new "lazy" instance definition, that defers coercions wherever possible. The advantage of the lazy instance definition over Mitchell's is that its use leads to an extremely compact representation for the unique minimal form of a typing. Following the framework described above, we describe a transformation, based on the lazy instance definition, that eliminates certain redundancies from typings. For many programs, removing redundancies from typings under the lazy instance definition leads to a coercion set of size at most one.

\subsection{Computing Unique Principal Types}

Previous work [Mit84,FM88] has shown that in the presence of subtypes, both the notion of type and the definition of instance must be suitably generalized. Thus, a type is written as a combination of a coercion set and type expression pair $(C, t)$. The instance relation on typings $C, A \vdash N: t$ is correspondingly generalized: typing $C^{\prime}, A^{\prime} \vdash N: t^{\prime}$ is an instance of $C, A \vdash N: t$ iff there exists a substitution $S$ such that $A^{\prime}=S(A), t^{\prime}=S(t)$ and $C^{\prime} \mathbb{H} S(C)$.

We implemented two provably correct algorithms based on the above definitions [Mit84,FM88]. Each algorithm produced a different type for the same program! While the types produced were consistent with each other, each being an instance of the other, this was clearly unsatisfactory. Upon examination, we discovered that the instance relation as defined above is exactly a preorder. Thus, while programs do possess principal types, the principal type is unique only up to equivalence under the instance relation. This explained the phenomenon of "multiple" principal types.

"Supported in part by NSF CCR-8706973 
Example 1 The following typings are both principal types for the identity function; it is important also to observe that $C_{1}$ is not equivalent to $C_{2}$.

$$
C_{1}=\{\alpha \triangleright \gamma, \gamma \triangleright \beta\}, \emptyset \vdash \lambda x . x: \alpha \rightarrow \beta, \quad C_{2}=\{\alpha \triangleright \beta\}, \emptyset \vdash \lambda x . x: \alpha \rightarrow \beta
$$

As the preorder nature of instance was not recognized in previous work [Mit84,FM88], the type inference algorithms described therein choose one possible representation for the principal type of a program. This explained why two different, correct, algorithms produced two different types. In addition we also found both representations unwieldy: The coercion set is large, 40-60 coercions for even toy programs, and has size proportional to program size. This effects the readability of typings: How does the user decipher the meaning of such a typing?, and efficiency of type inference: Checking consistency of a coercion set requires an algorithm quadratic in its size.

We solve both problems, lack of uniqueness and size, by providing a precise, technical definition of minimal typing. We prove that each equivalence class of typings possess a unique minimal representation. The main idea is that we can eliminate most type variables occurring in the coercion set which do not occur in the type assumption or inferred type. Simply removing all such type variables from a typing is unsound, as some may place constraints on the type assumption or inferred type, as in the example below.

\section{Example 2}

$$
n a p p l y \equiv \lambda f . \lambda x . \lambda n \text {.if } n=0 \text { then } x \text { else } f(\text { napply } f x n-1) .
$$

The principal typing for napply is:

$$
C, \emptyset \vdash \text { napply }:(\alpha \rightarrow \beta) \rightarrow\left(v_{x} \rightarrow\left(v_{n} \rightarrow v\right)\right)
$$

where $\left\{v_{x} \triangleright u, \beta \triangleright u, u \triangleright v, u \triangleright \alpha\right\}$ is a subset of $C$ and $u$ does not occur in the inferred type or type assumption. If we get rid of all the coercions involving $u$ we lose the constraint that in any type napply possesses, $\alpha$ and $v$ must have a common subtype, $u$, which is a common supertype of $v_{x}$ and $\beta$. $\square$

In practice, we find that it appears unnecessary to transform typings into their exact minimal representations. In its place, we provide a definition for a "redundant" type variable in coercion sets and show that the elimination of such variables must yield an equivalent typing. We show that the repeated elimination of "redundant" variables defines a function over typings and can therefore be used to transform typings. An algorithm that implements the function is described; the algorithm is cubic in the size of the original coercion set and reduces coercion set size to be proportional to the size of the type expression.

\subsection{A "lazy" instance definition}

To defer coercions occurring in typings, we introduce the following definition of lazy instance: typing $C^{\prime}, A^{\prime} \vdash N: t^{\prime}$ is an instance of $C, A \vdash N: t$ iff there exists substitution $S$ such that $C^{\prime} \Vdash S(C), C^{\prime} \Vdash A^{\prime} \triangleright$ $S(A), C^{\prime} \Vdash S(t) \triangleright t^{\prime}$. Our definition is a strict extension of the original; the difference lies in allowing the use of the information describing the context encoded in the coercion set $C^{\prime}$ to mediate the relationship between typings and in weakening the relationship between types from equality to inclusion $(D)$. As a consequence, many typings which were not previously instance related become instance related. Following the discussion above, we have not found it necessary to transform typings to exact minimal forms based on our instance relation. Instead, we use two transformations on typings that map most typings into a minimal form with respect to lazy instance. We describe these transformations below. 


\subsubsection{Cycle Elimination}

Mitchell's definition of type inclusion permits subtype symmetry: two distinct types, $t_{1}$ and $t_{2}$, may contain each other. As a consequence coercion sets often contain cycles of inter-related type variables and constants. Under our lazy instance relation we can equate all the participants in such a cycle and derive a much simpler but equivalent typing. Such a transformation cannot be proven correct using Mitchell's notion of instance, even if we restrict the definition of type inclusion to be anti-symmetric.

Example 3 Let $C_{1}=\{\alpha \triangleright \beta, \beta \triangleright \alpha\}, A_{1} \vdash N: t_{1}=\alpha \rightarrow \beta$ and $C_{2}=[\alpha / \beta]\left(C_{1}\right), A_{2}=[\alpha / \beta]\left(A_{1}\right) \vdash N$ : $t_{2}=[\alpha / \beta]\left(t_{1}\right)$ be typings. It is easy to see that $C_{2}, A_{2} \vdash N: t_{2}$ is an instance of $C_{1}, A_{1} \vdash N: t_{1}$ w.r.t. the original definition; however the opposite is not true as we cannot find substitution $S$ such that $t_{1}=S\left(t_{2}\right)$. It is not difficult to see that the two typings are equivalent with respect to our lazy instance definition. $\square$

\subsubsection{Eliminating Unnecessary Coercions under lazy Instance}

The principal type of a program $P$, as standardly derived, contains many coercions that can be deferred to the context in which $P$ is actually used.

\section{Example 4}

$$
\operatorname{comp} \equiv \lambda f \cdot \lambda g \cdot \lambda x \cdot f(g(x))
$$

The principal type:

$$
\{\kappa \triangleright \alpha, \sigma \triangleright \gamma, \beta \triangleright \rho\}, \emptyset \vdash \operatorname{comp}:(\alpha \rightarrow \beta) \rightarrow(\gamma \rightarrow \kappa) \rightarrow(\sigma \rightarrow \rho)
$$

In our approach, all the coercions in the type of comp may be deferred to the context of its use. Hence, its type is

$$
\{\}, \emptyset \vdash \text { comp: }(\alpha \rightarrow \beta) \rightarrow(\gamma \rightarrow \alpha) \rightarrow(\gamma \rightarrow \beta)
$$

When comp is used in expression comp $M$, we "recover" the lost coercion by permitting the type of $M$ to be a subtype of $(\alpha \rightarrow \beta)$. In this manner, all the coercions in the original typing for comp are recovered when comp is actually used. Thus, the only coercions that must appear in a typing for a term $N$ are those which are "internal" and cannot be deferred to the context in which $N$ is used.

Following the treatment of redundancy removal given for Mitchell's instance relation, we define a transformation on typings that removes redundancies from typings with respect to our lazy instance definition. A definition of redundant type variable is given and we prove that their repeated elimination defines a function over typings. We have found that for programs without multiple occurrences of lambda-bound names in widely differing contexts, the use of our lazy instance definition yields a coercion set of size at most one. The coercion set usually involves only type variables that occur in the type expression. Some examples of programs with non-trivial typings may be found in section 6 .

\section{Preliminaries}

Value expressions $N$ and type expressions $t$ are defined by the following abstract syntax.

$$
\begin{aligned}
& N:=c|x| \lambda x . N \mid N_{1} N_{2} \\
& t::=g_{c}\left|t_{1} \rightarrow t_{2}\right| \alpha
\end{aligned}
$$


A type expression $t$ that contains no type variable $\alpha$ is called a monotype; other type expressions are called polytypes. A general coercion is an ordered pair of types written $t_{1} \triangleright t_{2} ;$ a coercion is an ordered pair of types $t_{1} \triangleright t_{2}$, where both $t_{1}$ and $t_{2}$ are atomic types $\left(g_{c}, \alpha\right)$. A (general) coercion set $C=\left\{t_{i} \triangleright r_{i}\right\}$ is a set of (general) coercions. By types $(C)$ we will mean the set of types occurring in coercion set $C$. A substitution $S$ is a map from type variables to type expressions that is not equal to the identity function at only finitely many type variables. $\left[t_{1} / \alpha_{1}, \ldots, t_{n} / \alpha_{n}\right]$ is the substitution that maps each $\alpha_{i}$ to $t_{i}$ and is otherwise equal to the identity function. A type assumption $A$ is a finite map from value variables to type expressions. By $A ; \bar{x}: \bar{s}$ we mean a type assumption identical to $A$, except that it maps value variable $x_{i}$ to type expression $s_{i}$. If $\operatorname{domain}(A)=\operatorname{domain}\left(A^{\prime}\right)$, by $A \triangleright A^{\prime}$ we mean the coercion set $\left\{A(x) \triangleright A^{\prime}(x) \mid x \in \operatorname{domain}(A)\right\}$. A typing statement is written $C, A \vdash N: t$. The type inference rules are given by:

\begin{tabular}{|ll|}
{$[$ VAR $]$} & $C, A \vdash x: A(x)$ \\
{$[C O N S T A N T]$} & $C, A \vdash c: g_{c}$ \\
{$[A P P L Y]$} & $\frac{C, A \vdash N_{1}: t_{1} \rightarrow t_{2}, N_{2}: t_{1}}{C, A \vdash N_{1} N_{2}: t_{2}}$ \\
{$[A B S]$} & $\frac{C, A ; x: t_{1} \vdash N: t_{2}}{C, A \vdash \lambda x \cdot N: t_{1} \rightarrow t_{2}}$ \\
{$[C O E R C E]$} & $\frac{C, A \vdash N: t_{1}, C \Vdash\left\{t_{1} \triangleright t_{2}\right\}}{C, A \vdash N: t_{2}}$ \\
\hline
\end{tabular}

where the entailment relation $\|$ between coercion sets is defined by the following rules:

\begin{tabular}{|c|c|c|c|}
\hline$[A X I O M]$ & $C \cup\left\{t_{1} \triangleright t_{2}\right\} \mid-\left\{t_{1} \triangleright t_{2}\right\}$ & {$[$ CONST] } & $C \|\left\{g_{c_{i}} \triangleright g_{c_{k}}\right\}$ \\
\hline$[T R A N S]$ & $\frac{C \Downarrow-\left\{t_{1} \triangleright t_{2}, t_{2} \triangleright t_{3}\right\}}{C \|-\left\{t_{1} \triangleright t_{3}\right\}}$ & {$[R E F L E X]$} & $C \Vdash\{t \triangleright t\}$ \\
\hline$[A N D]$ & $\frac{C\left\|C_{1} \wedge C\right\| C_{2}}{C \| C_{1} \cup C_{2}}$ & {$[A R R O W]$} & $\frac{C \|-\left\{t_{1} \triangleright t_{1}^{\prime}, t_{2}^{\prime} \triangleright t_{2}\right\}}{C \|-\left\{t_{1}^{\prime} \rightarrow t_{2}^{\prime} \triangleright t_{1} \rightarrow t_{2}\right\}}$ \\
\hline
\end{tabular}

Since the set of coercions entailed by a coercion set is transitive-closed, thanks to [TRANS] rule, we can always replace a sequence of [COERCE] steps in a proof of a typing statement by a single [COERCE] step. This suggests the following characterization of the set of provable typing statements:

A typing statement $C, A \vdash N: p$ is a typing if

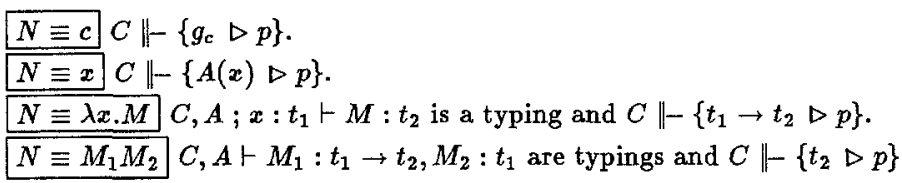

If $C, A \vdash N: t$ is a typing, the following specializations of $C, A \vdash N: t$ are also typings:

- $\gamma(C), \gamma(A) \|-N: \gamma(t)$, where $\gamma$ is a substitution.

- $C^{\prime}, A \vdash N: t$, where $C^{\prime} \Vdash C$.

- $C, A^{\prime} \vdash N: t$, where $C \|-\left.\left.A^{\prime}\right|_{F V(N)} \triangleright A\right|_{F V(N)}$.

- $C, A \vdash N: t^{\prime}$, where $C \|\left\{t \triangleright t^{\prime}\right\}$. 
That the first two specializations are typings is obvious. The other two cases are also true because we can always expand inferred type and type assumption by [COERCE] steps. Therefore, the typing $C, A \vdash N: t$ can be considered as more general than any of its specializations. By combining the specializations shown above, we define the instance ordering on typings as follows:

Definition 1 The typing $C^{\prime}, A^{\prime} \vdash N: t^{\prime}$ is an instance of the typing $C, A \vdash N: t$, written as $C, A \vdash N: t$ $\preceq C^{\prime}, A^{\prime} \vdash N: t^{\prime}$, if $\exists$ substitution $\gamma$ such that:

- $C^{\prime} \Vdash \gamma(C)$.

- $\left.\left.C^{\prime} \mathbb{F} A^{\prime}\right|_{F V(N)} \triangleright \gamma(A)\right|_{F V(N)}$.

- $C^{\prime} \Vdash \gamma(t) \triangleright t^{\prime}$.

Lemma 1 Typings are closed under the instance relation; i.e. if $C, A \vdash N: t$ is a typing then so is every instance $C^{\prime}, A^{\prime} \vdash N: t^{\prime}$.

Proof: Given above.

Since a typing can be considered as denoting all its instances, it's natural to regard two typings equivalent if they are instances of each other. More precisely, $C_{1}, A_{1} \vdash N: t_{1} \cong C_{2}, A_{2} \vdash N: t_{2}$ iff $C_{1}, A_{1} \vdash N: t_{1} \preceq C_{2}, A_{2} \vdash N: t_{2}$ and $C_{2}, A_{2} \vdash N: t_{2} \preceq C_{1}, A_{1} \vdash N: t_{1}$.

A typing statement $C, A \vdash N: p$ is a normal typing if

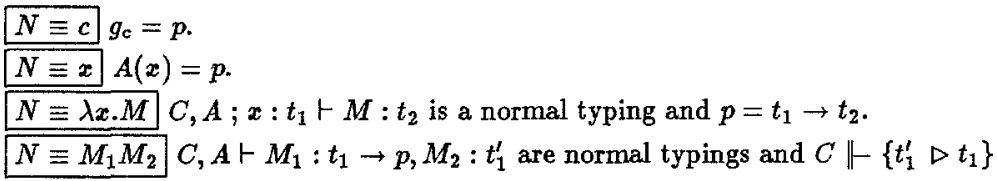

The idea behind normal typing is that the only essential coercions in a typing are those at application terms $M_{1} M_{2}$. This corresponds to our intuition that it's enough only to coerce values passed as arguments to functions. Our definition of normal typing is also the basis for algorithm TYPE in section 3 in which we carry out coercion steps only at argument subterm of function application during type inference. The following lemma justifies this intuition.

Lemma 2 Let $C, A \vdash N: t$ be a typing. Then there exists a normal typing $C^{\prime}, A^{\prime} \vdash N: t^{\prime}$ such that $C^{\prime}, A^{\prime} \vdash N: t^{\prime} \preceq C, A \vdash N: t$.

Proof: By structural induction on $\mathrm{N}$.

\section{Algorithm TYPE}

We say general coercion $t_{1} \triangleright t_{2}$ is matching if $t_{1}$ and $t_{2}$ are either both atomic types or both function types with matching range and domain. In previous work [FM88], we have described algorithm MATCH together with its correctness proof. Given a general coercion set $C$, algorithm MATCH either finds the most general substitution $S$ such that every coercion in $S(C)$ is matching or else it fails. If MATCH fails, no substitution that renders each coercion in $C$ matching exists. In [FM88] we have also described algorithm SIMPLIFY, which maps a matching coercion set $C$ into an equivalent set $C^{\prime}$ consisting only of coercions between atomic types (constants, variables). 
Input $C, A, N \in$ Coercion Set $\times$ Type Assumption $\times$ Value Expression

where $F V(N) \subseteq \operatorname{domain}(A)$.

Output $C^{\prime}, S, t \in$ Coercion Set $\times$ Substitution $\times$ Type Expression

such that $C^{\prime}, S(A) \vdash N: t$ is a typing.

$T Y P E(C, A, N)=$ case $N$ of

$$
N \equiv c
$$

$$
\begin{aligned}
& N \equiv x^{\left(C, I d, g_{c}\right)} \\
& N \equiv \lambda x \cdot M
\end{aligned}
$$

let $\left(C^{\prime}, S, t\right)=T Y P E(C, A ; x: \alpha, M)$

where $\alpha$ is a fresh type variable,

in $\left(C^{\prime}, S, S(\alpha) \rightarrow t\right)$

$N \equiv M_{1} M_{2}$

let $\left(C_{1}, S_{1}, t_{1}\right)=T Y P E\left(C, A, M_{1}\right)$;

$\left(C_{2}, S_{2}, t_{2}\right)=\operatorname{TYPE}\left(C_{1}, S_{1}(A), M_{2}\right)$;

$R=\operatorname{UNIFY}\left(S_{2}\left(t_{1}\right), \alpha \rightarrow \beta\right)$, where $\alpha, \beta$ are new

$R^{\prime}=M A T C H\left(R C_{2} \cup\left\{R\left(t_{2}\right) \triangleright R(\alpha)\right\}\right) ;$

$C^{\prime}=S I M P L I F Y\left(R^{\prime} R C_{2} \cup\left\{R^{\prime} R\left(t_{2}\right) \triangleright R^{\prime} R(\alpha)\right\}\right)$,

in

$\left(C^{\prime}, R^{\prime} R S_{2} S_{1}, R^{\prime} R(\beta)\right)$

Figure 1: Algorithm TYPE 
It is useful to compare the algorithm in Figure 1 with Mitchell's algorithm TYPE in [Mit84]. Mitchell's algorithm does not incorporate a type assumption (A above) that is "shared" between subexpressions of an expression; instead, in an application $M_{1} M_{2}$ both $M_{1}$ and $M_{2}$ are typed independently using independent type assumptions $A_{1}$ and $A_{2}$, which must later be reconciled using unification and matching. This may prove to be expensive as the size of a type assumption can be quite large in practice as it is bounded only by the total number of names defined in a program.

Theorem 1 TYPE is Sound

$\left(C^{\prime}, S, t\right)=T Y P E(C, A, N)$ succeeds $\Longrightarrow C^{\prime} \Vdash S(C) \wedge C^{\prime}, S(A) \vdash N: t$ is a typing.

Proof: See appendix.

Theorem 2 TYPE is Complete

If $C_{*}, A_{*} \vdash N: t_{*}$ is a typing and $\exists T$ s.t. $C_{*} \|-\left.T(C) \wedge A_{*}\right|_{F V(N)}=\left.T(A)\right|_{F V(N)}$ then $\left(C^{2}, S, t\right)=$ $T Y P E(C, A, N)$ succeeds and $C_{*}, A_{*} \vdash N: t_{*}$ is an instance of $C^{\prime}, S(A) \vdash N: t$.

Proof: See appendix. $\square$

\subsection{Consistency checking}

We say coercion set $C$ is consistent if $C \|-g_{c_{i}} \triangleright g_{c_{k}} \Rightarrow \emptyset \Vdash-g_{c_{i}} \triangleright g_{c_{k}}$. Expressed in words: a coercion set is consistent if every monotype coercion consequence is "true". From practical point of view, typings with inconsistent coercion set gives no information and hence should be excluded. Consistency checking can be implemented by examining the set of monotypes reachable from each monotype contained in coercion set $C$. Define a well-typing to be a typing $C, A \vdash N: t$, where $C$ is consistent. How do we compute well-typings? We simply run algorithm TYPE and check the final coercion set for consistency. If it is consistent, we are done; otherwise, we fail and conclude that no well-typing exists:

let $\left(C^{\prime}, S, t\right)=T Y P E(C, A, N)$ in

if $C^{t}$ is consistent then $\left(C^{\prime}, S, t\right)$ such that $C^{\prime}, S(A) \vdash N: t$ is a well-typing

else fail

That this method is sound is obvious; its completeness is argued as follows: To see that the above is complete, we need to consider two cases. For the first, we have that $C^{\prime}$ is consistent. But then, the syntactic completeness of TYPE ensures the syntactic completeness of the above algorithm. For the second case, let $C^{\prime}$ be inconsistent. We will argue that no well-typing $C_{*}, A_{*} \vdash N: t_{*}$ exists. Assume otherwise; as TYPE is syntactically complete we can find substitution $\gamma$ with $C_{*} \| \gamma\left(C^{\prime}\right)$. Now, since $C_{*}$ is consistent we must have that $\gamma\left(C^{\prime}\right)$ is consistent. But then $C^{\prime}$ must be consistent as well and we have arrived at a contradiction.

\section{Computing Principal Types}

\subsection{Existence and Uniqueness}

The elimination of type variables from the coercion set is based on the following insight: for any typing, types occurring in the coercion set component can be partitioned into two classes: those that are visible or observable in the sense future coercions may refer to them; Types that are not visible will never be involved in any future coercions. All type constants are observable, as are type variables that occur either 
in the type assumption or the inferred type. All other types occurring in the coercion set are not visible. For example, consider the typing:

$$
\{v \triangleright \alpha, v \triangleright \eta, \alpha \triangleright \text { int }, \beta \triangleright \gamma, \beta \triangleright \alpha\}, \emptyset \vdash N: \alpha \rightarrow \eta
$$

then $\alpha, \eta$, int are observable and $v, \beta, \gamma$ are not.

Intuitively speaking, type variables that are not observable are useless unless they constrain observable variables by "connecting" them together. In the example above $\beta$ and $\gamma$ are useless and we could dispense completely with the coercions $\beta \triangleright \alpha$ and $\beta \triangleright \gamma$ and arrive at a smaller and equivalent coercion set. However, although $v$ is not observable we can not get rid of the coercions $v \triangleright \alpha$ and $v \triangleright \eta$ which involve $v$.

Following the discussion above, define $\operatorname{Obv}(C, A \vdash N: t)$ to be the set of type variables occuring in $A$ or $t$ and let $\operatorname{Intv}(C, A \vdash N: t)$ be $\operatorname{Vars}(C)-O b v(C, A \vdash N: t)$. A substitution $S$ is a renaming on the set $V$ of type variables if the restriction of $S$ to $V$ is one to one. Similarly, $S$ is an identity on $V$ if the restriction of $S$ to $V$ is an identity.

Definition 2 A typing $C, A \vdash N: t$ is minimal iff $S$ is an identity on $O b v(C, A \vdash N: t) \wedge C \|-S(C) \Longrightarrow$ $S$ is a renaming on type variables in $C, A \vdash N: t$. A typing is redundant if it is not minimal.

Observe that whenever $C \|-S(C), S$ must map variables in $\operatorname{Intv}(C, A \vdash N: t)$ to either the variables in $\operatorname{Vars}(C)$ or type constants. Further, without loss of generality we can assume $S$ is an identity on variables outside $\operatorname{Vars}(C)$. Define $S u b s(C, A \vdash N: t)$ to be the set of substitutions $S$ where:

$$
S(v)= \begin{cases}v, & v \notin \operatorname{Intv}(C, A \vdash N: t) \\ \text { a type constant or a variable } \in \operatorname{Vars}(C), & v \in \operatorname{Intv}(C, A \vdash N: t)\end{cases}
$$

It's not difficult to see that $S u b s(C, A \vdash N: t)$ is finite, as the number of distinct variables in a typing is finite. Thus the minimality of a typing can be decided by checking the condition $C \Downarrow-S(C)$ for all substitution $S \in S u b s(C, A \vdash N: t)$. The following algorithm computes minimal typings:

$\operatorname{minimize}(C, A \vdash N: t)=$

if $\exists S \in S u b s(C, A \vdash N: t)$ such that $C \Vdash S(C) \wedge S$ not a renaming on $\operatorname{Intv}(C, A \vdash N: t)$

then minimize $(S(C, A \vdash N: t))$

else $C, A \vdash N: t$

Since $\operatorname{Subs}(C, A \vdash N: t)$ is finite and checking the entailment between two finite coercion sets is decidable the "if" condition can be effectively evaluated. Since the substitution satisfying the "if" condition must remove at least one variable in $\operatorname{Intv}(C, A \vdash N: t),|\operatorname{Intv}(C, A \vdash N: t)|$ is strictly decresing. Therefore minimize always terminates and returns a minimal typing as its final result. This proves the existence of minimal typings.

Let $C$ be an acyclic coercion set. By $C^{*}$ we mean the reflexive, transitive closure of $C$. It's obvious that $C^{*}$ exactly consists of the atomic coercions entailed by $C$. More precisely:

$$
C \| a \triangleright a^{\prime} \text { iff } a \triangleright a^{\prime} \in C^{*}
$$

A coercion set $C$ is equivalent to the coercion set $C^{\prime}$, written $C \equiv C^{\prime}$, iff $C \|-C^{\prime}$ and $C^{\prime} \Vdash C$ or, equivalently, $C^{*}=C^{\prime *}$. The equivalence of two coercion sets is preserved under substitution.

\section{Theorem 3 Minimal Typing Is Unique}

If $C_{1}, A_{1} \vdash N: t_{1} \cong C_{2}, A_{2} \vdash N: t_{2}$ are minimal typings then there exists a renaming $S$ such that: 
- $t_{1}=S\left(t_{2}\right)$.

- $\left.A_{1}\right|_{F V(N)}=S\left(\left.A_{2}\right|_{F V(N)}\right)$.

- $C_{1} \equiv S\left(C_{2}\right)$.

Proof: By definition of $\cong$ there exists $S_{1}, S_{2}$ such that:

(1) $t_{1}=S_{1}\left(t_{2}\right)$ and $t_{2}=S_{2}\left(t_{1}\right)$.

(2) $\left.A_{1}\right|_{F V(N)}=S_{1}\left(\left.A_{2}\right|_{F V(N)}\right)$ and $\left.A_{2}\right|_{F V(N)}=S_{2}\left(\left.A_{1}\right|_{F V(N)}\right)$.

(3) $C_{1} \|-S_{1}\left(C_{2}\right)$ and $C_{2} \|-S_{2}\left(C_{1}\right)$.

By (1) and (2) we have $t_{2}=S_{2} S_{1}\left(t_{2}\right)$ and $\left.A_{2}\right|_{F V(N)}=S_{2} S_{1}\left(\left.A_{2}\right|_{F V(N)}\right)$ and hence $S_{2} S_{1}$ must be an identity on $\operatorname{Var}\left(t_{2}\right) \cup \operatorname{Var}\left(\left.A_{2}\right|_{F V(N)}\right)$. This in turn entails that $S_{1}$ is a renaming on $\operatorname{Var}\left(t_{2}\right) \cup \operatorname{Var}\left(\left.A_{2}\right|_{F V(N)}\right)$.

We now proceed to show $S_{1}$ is also a renaming on $\operatorname{Var}\left(C_{2}\right)-\left(\operatorname{Var}\left(t_{2}\right) \cup \operatorname{Var}\left(\left.A_{2}\right|_{F V(N)}\right)\right)$ and $C_{1} \equiv$ $S_{1}\left(C_{2}\right)$. By (3) we have $C_{2} \Vdash S_{2} S_{1}\left(C_{2}\right)$. Since $C_{2}, A_{2} \vdash N: t_{2}$ is minimal, by the definition of minimality of typings $S_{2} S_{1}$ is a renaming on $\operatorname{Var}\left(C_{2}\right)-\left(\operatorname{Var}\left(t_{2}\right) \cup \operatorname{Var}\left(\left.A_{2}\right|_{F V(N)}\right)\right)$ and hence $S_{1}$ is a renaming on $\operatorname{Var}\left(C_{2}\right)-\left(\operatorname{Var}\left(t_{2}\right) \cup \operatorname{Var}\left(\left.A_{2}\right|_{F V(N)}\right)\right)$. By the same argument we have $S_{2}$ is a renaming on $\operatorname{Var}\left(C_{1}\right)-$ $\left(\operatorname{Var}\left(t_{1}\right) \cup \operatorname{Var}\left(\left.A_{1}\right|_{F V(N)}\right)\right)$. Since $C_{1} \| S_{1}\left(C_{2}\right), C_{1}^{*} \supseteq\left(S_{1}\left(C_{2}\right)\right)^{*}$ and hence $\left|C_{1}^{*}\right| \geq\left|\left(S_{1}\left(C_{2}\right)\right)^{*}\right|$. Since renaming preserves the cardinality of coercion sets we can use (3) to get the fact that $\left|\left(S_{1}\left(C_{2}\right)\right)^{*}\right|=\mid$ $\left(C_{2}\right)^{*}|\geq|\left(S_{2}\left(C_{1}\right)\right)^{*}|=| C_{1}{ }^{*} \mid$. Combining the above results we conclude that $C_{1}{ }^{*}=\left(S_{1}\left(C_{2}\right)\right)^{*}$, as $\left|C_{1}{ }^{*}\right|=$ $\left(S_{1}\left(C_{2}\right)\right)^{*} \mid$ and $C_{1}{ }^{*} \supseteq\left(S_{1}\left(C_{1}\right)\right)^{*}$, and hence $C_{1} \equiv S_{1}\left(C_{2}\right)$. Let $S$ be $S_{1}$ then we are done.

\subsection{Removing Redundancy from Typings}

By Theorem 3, eliminating redundancy in two equivalent typings ends up with the same minimal typing up to renaming of type variables and the equivalence of coercion sets. This suggests a unique representative for equivalent typings and thus the preorder on typings can be replaced by a partial order on minimal typings.

In practice, we have not found it necessary to compute exact minimal typings. There are two reasons for this: first, the computation of such a form appears to require exhaustive checking of the condition $C \Vdash S(C)$ for all $S \in S u b s(C, A \vdash N: t)$; second, we find most redundancy is of a simple form which can be efficiently detected and removed.

Let $C$ be a coercion set and $a$ be an atomic type in $C$. Define:

$$
\begin{aligned}
& \text { above }_{C}(a)=\left\{a^{\prime} \mid a \triangleright a^{\prime} \in C^{*}\right\} \\
& \text { below }_{C}(a)=\left\{a^{\prime} \mid a^{\prime} \triangleright a \in C^{*}\right\}
\end{aligned}
$$

Definition 3 Variable $\alpha \in$ types $(C)$, is G-subsumed by $\beta \in \operatorname{types}(C)$ in $C$, written $\alpha \leq_{G} \beta$ in $C$, if

1. above $_{C}(\alpha)-\{\alpha\} \subseteq$ above $_{C}(\beta)$.

2. below $_{C}(\alpha)-\{\alpha\} \subseteq$ below $_{C}(\beta)$.

In the following lemma, we show that if $\alpha \leq_{G} \beta$ in $C$ then we can identify $\alpha$ with $\beta$ without affecting any coercion consequence of $C$ which does not involve $\alpha$.

Lemma 3 If $\alpha \leq_{G} \beta$ in $C$ and $C^{\prime}=[\beta / \alpha](C)$ then:

$$
C\left\|-a \triangleright a^{\prime} \wedge a \neq \alpha \wedge a^{\prime} \neq \alpha \Longleftrightarrow C^{\prime}\right\|-a \triangleright a^{\prime}
$$




\section{Proof:}

$(\Longrightarrow)$ :

Since $C \|-a \triangleright a^{\prime}, a \triangleright a^{\prime} \in C^{*}$. Hence $a \triangleright a^{\prime} \in[\beta / \alpha]\left(C^{*}\right)$. Since $C \equiv C^{*}$ and $\equiv$ is preserved under substitution, $C^{\prime}=[\beta / \alpha] \equiv[\beta / \alpha]\left(C^{*}\right)$ and hence $C^{\prime} \Vdash a \triangleright a^{\prime}$.

$(\Longleftarrow)$ :

Let Deleted_coercions $=\left\{a \triangleright a^{\prime} \in C \mid a=\alpha\right.$ or $\left.a^{\prime}=\alpha\right\}$ and Added_coercions $=\{a \triangleright \beta \mid a \triangleright \alpha \in$ $C\} \cup\{\beta \triangleright a \mid \alpha \triangleright a \in C\}$. Observe that $C^{\prime}=(C \cup$ Added_coercions $)-$ Deleted_coercions. Since $\alpha \leq_{G} \beta$ in $C$, the coercions in Added_coercions is actually entailed by $C$. Therefore, the set of coercions entailed by $C^{\prime}$ is a subset of that entailed by $C$.

The following consequences of Lemma 3 will be used frequently in the rest of this section:

fact1 if $\alpha \leq_{G} \beta$ in $C$ then $C \|[\beta / \alpha](C)$.

fact 2 if $\alpha \leq_{G} \beta$ in $C$ and $C^{\prime}=[\beta / \alpha](C)$ then $\forall a \in C^{\prime}$, above $C^{\prime}(a)=$ above $_{C}(a)-\{\alpha\} \wedge$ below $_{C^{\prime}}(a)=$ below $w_{C}(\alpha)-\{\alpha\}$.

fact3 Let $C \equiv C^{\prime}, \alpha \leq_{G} \beta$ in $C$, and $\alpha \leq_{G} \beta^{\prime}$ in $C^{\prime}$, then $[\beta / \alpha](C) \equiv\left[\beta^{\prime} / \alpha\right]\left(C^{\prime}\right)$.

Define the relation $\mapsto_{G}$ on typings as follows:

$$
C, A \vdash N: t \mapsto_{G} C^{\prime}, A \vdash N: t \Longleftrightarrow\left\{\begin{array}{l}
(1) \alpha \leq_{G} \beta \text { in } \mathrm{C} . \\
(2) \alpha \operatorname{not} \text { in } A \text { or } t . \\
(3) C^{\prime}=[\beta / \alpha](C) .
\end{array}\right.
$$

Let $C, A \vdash N: t \mapsto_{G} C^{\prime}, A \vdash N: t$ by $[\beta / \alpha]$ and $C^{\prime}=[\beta / \alpha](C)$. We show that $C, A \vdash N: t \cong$ $C^{\prime}, A \vdash N: t$. That $C^{\prime}, A \vdash N: t$ is an instance of $C, A \vdash N: t$ is obvious. To show the opposite direction observe, by fact 1 , that we have $C \|-[\beta / \alpha](C)$, hence $C, A \vdash N: t$ is also an instance of $[\beta / \alpha](C), A \vdash N: t$. This result can be extended to any sequence of $\mapsto_{G}$ steps. Therefore, $C, A \vdash N: t \stackrel{{ }^{*}}{{ }_{G}} C^{\prime}, A \vdash N: t$ implies $C^{\prime}, A \vdash N: t \cong C, A \vdash N: t$.

For our purposes, the most important property of $\mapsto_{G}$ is that it defines a function on typings up to the equivalence of coercion sets and thus maps each typing to a unique equivalent typing without "useless" variables.

Lemma 4 Let $C \equiv \bar{C}$.

$$
\begin{aligned}
& \left\{\begin{array}{llll}
C, A \vdash N: t & \mapsto G & C_{1}, A \vdash N: t & b y\left[b_{1} / a_{1}\right] \\
\bar{C}, A \vdash N: t & \mapsto G & C_{2}, A \vdash N: t & \text { by }\left[b_{2} / a_{2}\right]
\end{array}\right\} \Longrightarrow \exists C^{\prime} \equiv \bar{C}^{\prime}, \text { such that: } \\
& \left\{\begin{array}{lll}
C_{1}, A \vdash N: t & \stackrel{*}{\mapsto}_{G} & C^{\prime}, A \vdash N: t \\
C_{2}, A \vdash N: t & \stackrel{*}{\mapsto}_{G} & \bar{C}^{\prime}, A \vdash N: t
\end{array}\right\}
\end{aligned}
$$

\section{Proof:}

$a_{1} \neq b_{2}$ and $a_{2} \neq b_{1}$ :

If $a_{1}=a_{2}$ then by fact3 of lemma $3 C_{1} \equiv C_{2}$ and no further reduction sequences are needed to reconcile $C_{1}, A \vdash N: t$ and $C_{2}, A \vdash N: t$. Assume otherwise, then by fact2 the subsumption relation between $a_{1}$ and $b_{1}$ in $C$ (resp. $a_{2}$ and $b_{2}$ in $\bar{C}$ ) is not effected by the substitution $\left[b_{2} / a_{2}\right]$ (resp. $\left[b_{1} / a_{1}\right]$ ). Hence we can apply the one-step reduction $\left[b_{2} / a_{2}\right]$ to $C_{1}, A \vdash N: t$ and the one-step reduction $\left[b_{1} / a_{1}\right]$ to $C_{2}, A \vdash N: t$. By fact2 this reconciles $C_{1}, A \vdash N: t$ and $C_{2}, A \vdash N: t$. 


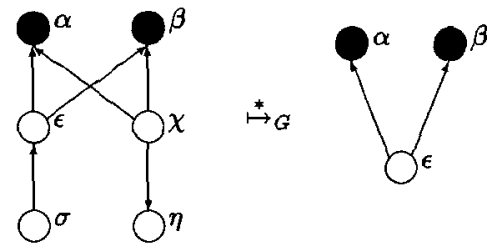

Figure 2

$a_{1} \neq b_{2}$ and $a_{2}=b_{1}:$

Since $a_{1} \leq_{G} b_{1}$ and $b_{1}=a_{2} \leq_{G} b_{2}$, by transitivity of $\leq_{G}, a_{1} \leq_{G} b_{2}$. By fact2 the subsumption relation between $a_{1}$ and $b_{2}$ in $\bar{C}$ (resp. $a_{2}$ and $b_{2}$ in $C$ ) is not effected by the substitution $\left[b_{2} / a_{2}\right]$ (resp. $\left[b_{1} / a_{1}\right]$ ). Therefore we can apply the one-step reduction $\left[b_{2} / a_{1}\right]$ to $C_{2}, A \vdash N: t$ and apply the one-step reduction $\left[b_{2} / a_{2}\right]$ (or $\left[b_{2} / b_{1}\right]$ ) to $C_{1}, A \vdash N: t$. By fact2 this reconciles $C_{1}, A \vdash N: t$ and $C_{2}, A \vdash N: t$.

$a_{1}=b_{2}$ and $a_{2} \neq b_{1}:$ Same as previous case.

$a_{1}=b_{2}$ and $a_{2}=b_{1}$ :

Then $C_{1}$ is identical to $C_{2}$ up to variable renaming.

$\square$

Theorem 4 Every typing $C, A \vdash N: t$ has a unique normal form under $\mapsto_{G}$ up to variable renaming and the equivalence of coercion sets.

Proof: As $\mid$ types $\left(C_{i}\right) \mid$ is strictly decreased in any reduction sequence, no reduction sequence continues for ever. Hence the relation $\mapsto_{G}$ is strongly normalizing. By Proposition 3.1.25 in [Bar84] and lemma 4, $\mapsto_{G}$ is Church-Rosser up to the equivalence of coercion sets. Hence every typing has a normal form upto equivalence under $\mapsto G$.

Example 5 Let $C=\{\sigma \triangleright \epsilon, \epsilon \triangleright \alpha, \epsilon \triangleright \beta, \chi \triangleright \alpha, \chi \triangleright \beta, \chi \triangleright \eta\}$ be a coercion set where only $\alpha$ and $\beta$ occur in the inferred type or type assumption. The minimized form of $C$ is shown by Figure 2.

By the theorem above, $\mapsto G$ defines a function on typings up to the identification of equivalent coercion sets. In other words, starting from a given typing, different "maximal" sequences of $\mapsto_{G}$ steps all end up with typings with the same $A$ and $t$ components and equivalent but possibly different coercion set component. If it is so desired a unique coercion set can always be derived by replacing the transformed coercion set by its transitive reduction.

We implement $\mapsto_{G}$ in the following manner: we top-sort the coercion set and compute above and below sets for vertices in two passes over the coercion set. Comparison of above and below can be implemented in constant time by using the standard bit-string implementation of finite (and small) sets. Finding a subsumable variable may take up to $n^{2}$ comparisons in an $n$ vertex graph; there are at most $n$-vertices in an $n$-vertex coercion set that can be G-subsumed by other vertices. Therefore, in worst case, the complexity is cubic in the size of the coercion set component. 


\section{Eliminating Unnecessary Coercions under lazy Instance}

The expand_polarity of a type variable $\alpha$ in a type $t$ is a subset of $\{\uparrow, \downarrow\}$ indicating the effect on occurrences of $\alpha$ in $t$ when $t$ is coerced into a super type $t^{\prime}$. There are four ways in which occurrences of $\alpha$ may be effected: no effect, expansion, contraction and a combination of expansion and contraction. These four possibilities are represented by $\emptyset,\{\uparrow\},\{\downarrow\}$, and $\{\uparrow, \downarrow\}$ respectively. The symmetric notion, contract_polarity, is defined similarly.

$$
\text { expand_polarity }(\alpha, t)= \begin{cases}\{\uparrow\} & \text { if } \alpha=t . \\ \text { contract_polarity }\left(\alpha, t_{1}\right) \cup \text { expand_polarity }\left(\alpha, t_{2}\right) & , \text { if } t=t_{1} \rightarrow t_{2} . \\ \emptyset & , O / W .\end{cases}
$$

and

$$
\text { contract_polarity }(\alpha, t)= \begin{cases}\{\downarrow\} & \text { if } \alpha=t . \\ \text { expand_polarity }\left(\alpha, t_{1}\right) \cup \text { contract_polarity }\left(\alpha, t_{2}\right) & \text {, if } t=t_{1} \rightarrow t_{2} . \\ \emptyset & , o / W .\end{cases}
$$

Observe that expand_polarity $(\alpha, t)=\{\uparrow\}(\{\downarrow\})$ iff contract_polarity $(\alpha, t)=\{\downarrow\}(\{\uparrow\})$. As examples, expand_polarity $(\alpha,(\alpha \rightarrow \beta) \rightarrow(\gamma \rightarrow \beta))=\{\uparrow\}$ and contract_polarity $(\alpha,(\alpha \rightarrow \beta) \rightarrow(\gamma \rightarrow \beta))=\{\downarrow\}$.

Definition 4 Let $\alpha \in$ types $(C)$ and $\alpha$ occurs in $t$. We say $\alpha$ is $S$-subsumed by $r$ in $C$ and $t$, written $\alpha \leq_{S} r$ in $C$ and $t$, iff either

1. $C \|-\{r \triangleright \alpha\}$ and expand_polarity $(\alpha, t)=\{\uparrow\}$ and below $(\alpha)-\{\alpha\} \subseteq$ below $_{C}(r)$.

or

2. $C \Vdash\{\alpha \triangleright r\}$ and expand_polarity $(\alpha, t)=\{\downarrow\}$ and above $C(\alpha)-\{\alpha\} \subseteq$ above $C(r)$.

Observe that $\leq_{S}$ is a special case of $\leq_{G}$ and hence lemma 3 also holds for $\leq_{S}$.

Let $C, A \vdash N: t$ be a typing and domain $(A)=\left\{x_{1}, \ldots, x_{n}\right\}$. By type_closure $(C, A \vdash N: t)$ we mean the type expression $A\left(x_{1}\right) \rightarrow \ldots \rightarrow A\left(x_{n}\right) \rightarrow t$. Define the relation $\mapsto_{S}$ on typings by the following:

$$
C, A \vdash N: t \mapsto_{S} C^{\prime}, A^{\prime} \vdash N: t^{\prime} \Longleftrightarrow\left\{\begin{array}{l}
\text { (1) } \alpha \leq_{S} \beta \text { in } C \text { and type_closure }(C, A \vdash N: t) \\
(2) C^{\prime}=[\beta / \alpha](C) \\
(3) A^{\prime}=[\beta / \alpha](A) \\
(4) t^{\prime}=[\beta / \alpha](t)
\end{array}\right.
$$

If $\alpha \leq_{S} \beta$ in $C$ and type_closure $(C, A \vdash N: t)$ then, without loss of generality, we can assume $C \sharp\{\beta \triangleright \alpha\}$ and expand_polarity $(\alpha$, type_closure $(C, A \vdash N: t))=\{\uparrow\}$. By the definition of expand_polarity, $\forall x \in$ $\operatorname{domain}(A)$, either contract_polarity $(\alpha, A(x))=\{\uparrow\}$ or contract_polarity $(\alpha, A(x))=\emptyset$. Since $C \|\{\beta \triangleright$ $\alpha\}, \forall x \in \operatorname{domain}(A), C \|\{A(x) \triangleright[\beta / \alpha](A(x))\} ;$ in other words, $C \|-A \triangleright[\beta / \alpha](A)$. Similarly, either expand_polarity $(\alpha, t)=\{\uparrow\}$ or expand_polarity $(\alpha, t)=\emptyset$; either entails that $C \|-\{[\beta / \alpha](t) \triangleright t\}$. Since lemma 3 holds for $\leq_{S}, C \Vdash[\beta / \alpha](C)$. Hence $C, A \vdash N: t$ is an instance of $[\beta / \alpha](C),[\beta / \alpha](A) \vdash N:[\beta / \alpha](t)$. This implies the equivalence of this two typings, as $[\beta / \alpha](C),[\beta / \alpha](A) \vdash N:[\beta / \alpha](t)$ is an instance of $C, A \vdash N: t$.

Following the development for $\mapsto_{G}$, we show that $\mapsto_{S}$ defines a function on typings, up to the equivalence of coercion sets.

Lemma 5 Let $C \equiv \bar{C}$ be acyclic.

$$
\left\{\begin{array}{cccc}
C, A \vdash N: t & \mapsto S & C_{1}, A_{1} \vdash N: t_{1} & \text { by }\left[b_{1} / a_{1}\right] \\
\bar{C}, A \vdash N: t & \mapsto S & C_{2}, A_{2} \vdash N: t_{2} & \text { by }\left[b_{2} / a_{2}\right]
\end{array}\right\} \Rightarrow \exists C^{\prime} \equiv \vec{C}^{\prime}, A^{\prime}, \text { and } t^{\prime} \text { such that : }
$$




$$
\left\{\begin{array}{lll}
C_{1}, A_{1} \vdash N: t_{1} & \stackrel{*}{\mapsto} S & C^{\prime}, A^{\prime} \vdash N: t^{\prime} \\
C_{2}, A_{2} \vdash N: t_{2} & \stackrel{*}{\mapsto} S & \bar{C}^{\prime}, A^{\prime} \vdash N: t^{\prime}
\end{array}\right\}
$$

Proof:

$a_{1} \neq b_{2}$ and $a_{2} \neq b_{1}$ :

If $a_{1}=a_{2}$ then either $C \|-\left\{a_{1} \triangleright b_{1}, a_{1} \triangleright b_{2}\right\}$ or $C \|-\left\{b_{1} \triangleright a_{1}, b_{2} \triangleright a_{1}\right\}$. This entails either $b_{1} \in$ above $_{C}\left(a_{1}\right)-\left\{a_{1}\right\} \subseteq$ above $_{C}\left(b_{2}\right) \wedge b_{2} \in$ above $_{C}\left(a_{1}\right)-\left\{a_{1}\right\} \subseteq$ above $_{C}\left(b_{1}\right)$ or $b_{1} \in$ below $_{C}\left(a_{1}\right)-\left\{a_{1}\right\} \subseteq$ below $_{C}\left(b_{2}\right) \wedge b_{2} \in$ below $_{C}\left(a_{1}\right)-\left\{a_{1}\right\} \subseteq$ below $w_{C}\left(b_{1}\right)$. Hence $b_{1}=b_{2}$, as $C$ is acyclic. Assume otherwise, by fact2 the subsumption relation between $a_{1}$ and $b_{1}$ in $\bar{C}$ (resp. $a_{2}$ and $b_{2}$ in $C$ ) is not effected by the substitution $\left[b_{2} / a_{2}\right]$ (resp. $\left[b_{1} / a_{1}\right]$ ). Therefore, we can apply the one-step reduction $\left[b_{2} / a_{2}\right]$ to $C_{1}, A_{1} \vdash N: t_{1}$ and the one-step reduction $\left[b_{1} / a_{1}\right]$ to $C_{2}, A_{2} \vdash N: t_{2}$. This reconciles $C_{1}, A_{1} \vdash N: t_{1}$ and $C_{2}, A_{2} \vdash N: t_{2}$.

$a_{1} \neq b_{2}$ and $a_{2}=b_{1}:$

If $C \|-\left\{a_{1} \triangleright b_{1}, b_{2} \triangleright b_{1}\right\}$ then $a_{1} \in$ below $_{C}\left(b_{1}\right)-\left\{b_{1}\right\} \subseteq$ below $_{C}\left(b_{2}\right)$. This entails $b_{2} \in a b o v e_{C}\left(b_{1}\right)-\left\{b_{1}\right\}$, as $b_{2} \in$ above $_{C}\left(a_{1}\right)-\left\{a_{1}\right\} \subseteq$ above $_{C}\left(b_{1}\right)$. Hence, $b_{1}=b_{2}$, as $C \|-\left\{b_{1} \triangleright b_{2}, b_{2} \triangleright b_{1}\right\}$ and $C$ is acyclic. The symmetric situation where $C \|-\left\{b_{1} \triangleright a_{1}, b_{1} \triangleright b_{2}\right\}$ can be proved similarly. The remaining possibilities are either $C \|-\left\{a_{1} \triangleright b_{1}, b_{1} \triangleright b_{2}\right\}$ or $C \|-\left\{b_{2} \triangleright b_{1}, b_{1} \triangleright a_{1}\right\}$ which entail $a_{1} \leq_{s} b_{2}$ in $\bar{C}$. By fact2, the subsumption relations between $a_{1}$ and $b_{2}$ in $\bar{C}$ (resp. $b_{1}=a_{2}$ and $b_{2}$ in $C$ ) is not effected by the substitution $\left[b_{2} / a_{2}\right]$ (resp. $\left.\left[a_{1} / b_{1}\right]\right)$. Therefore we can apply the one-step reduction $\left[b_{2} / a_{1}\right]$ to $C_{2}, A_{2} \vdash N: t_{2}$ and apply the one-step reduction $\left[b_{2} / a_{2}\right]\left(\right.$ or $\left.\left[b_{2} / b_{1}\right]\right)$ to $C_{1}, A_{1} \vdash N: t_{1}$. This reconciles $C_{1}, A_{1} \vdash e: t_{1}$ and $C_{2}, A_{2} \vdash N: t_{2}$.

$a_{1}=b_{2}$ and $a_{2} \neq b_{1}:$ Same as previous case.

$a_{1}=b_{2}$ and $a_{2}=b_{1}:$

Then $C_{1}$ is identical to $C_{2}, A_{1}$ is identical to $A_{2}$, and $t_{1}$ is identical to $t_{2}$, up to variable renaming.

Theorem 5 Every typing $C, A \vdash N: t$ has a unique normal form under $\mapsto S$ up to variable renaming and the equivalence of coercion sets.

Proof: Similar to theorem4.

The implementation of $\stackrel{*}{\rightarrow} S$ is almost the same as that of $\stackrel{*}{\mapsto}_{G}$. The only difference is checking the polarity when looking for a $S$-subsumed variable. This can be computed efficiently. In the following program we show the final typing of napply after carrying out coercion elimination:

Example 6 Consider program napply:

$$
n a p p l y=\lambda f . \lambda x . \lambda n . \text { if } n=0 \text { then } x \text { else napply } f(n-1)(f x)
$$

Algorithm TYPE followed by the minimizations described before results in the following type for napply:

$$
\{\eta \triangleright \alpha, \beta \triangleright \eta, \eta \triangleright \sigma, \gamma \triangleright \text { int }\}, \emptyset \vdash \text { napply }:(\alpha \rightarrow \beta) \rightarrow \eta \rightarrow \gamma \rightarrow \sigma
$$

after coercion elimination (shown in Figure 3) we arrive at the following type:

$$
\emptyset, \emptyset \vdash \text { napply }:(\eta \rightarrow \eta) \rightarrow \eta \rightarrow \text { int } \rightarrow \eta
$$




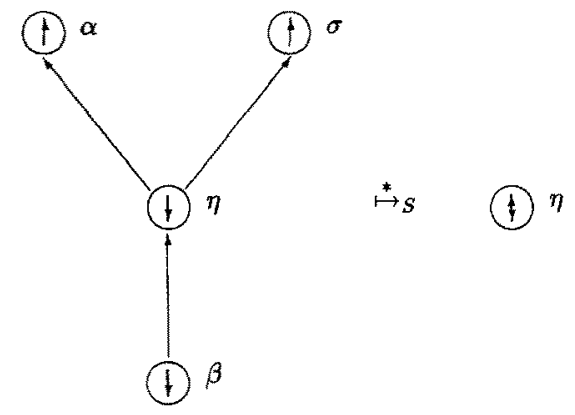

Figure 3

\section{Characterizing the shape of Coercion Sets}

For most programs, our techniques yield typings in which the coercion set component is at most of size one. However, it is not difficult to find examples that make full use of the power of the coercion set and type notation and where the size of the minimal coercion set is proportional to the size of the type expression component of the typing.

\section{Example 7}

$$
\{\eta \triangleright \alpha, \eta \triangleright \tau, \gamma \triangleright \tau, \sigma \triangleright \alpha\}, \emptyset \vdash \lambda f \cdot \lambda g \cdot \lambda x \cdot(f g x, g f x):(\alpha \rightarrow \gamma) \rightarrow(\tau \rightarrow \sigma) \rightarrow(\eta \rightarrow \gamma * \sigma)
$$

It is also possible to find examples of typings where the coercion set involves type variables that do not occur elsewhere in the typing in an essential way.

\section{Example 8}

$$
\{\eta \triangleright \alpha, \eta \triangleright \tau, \gamma \triangleright \tau\} \emptyset \vdash \lambda f . \lambda x . f s t(f x, \lambda g .(f g x, g f x)):(\alpha \rightarrow \gamma) \rightarrow(\eta \rightarrow \gamma)
$$

\section{References}

[Bar84] H. Barendregt. The Lambda Calculus, its Syntax and Semantics. 1984.

[FM88] Y-C Fuh and P Mishra. Type inference with subtypes. In ESOP-88 (Also Stony Brook TR 87/25), March, 1988.

[Mil78] Robin Milner. A theory of type polymorphism in programming. In JCSS 17, 1978.

[Mit84] J. C. Mitchell. Coercion and type inference. In POPL XI, 1984.

\section{Appendix I: TYPE is sound and complete}

\section{Theorem 6 TYPE is Sound}

$\left(C^{\prime}, S, t\right)=T Y P E(C, A, N)$ succeeds $\Longrightarrow C^{\prime} \Vdash S(C) \wedge C^{\prime}, S(A) \vdash N: t$ is a typing. 


\section{Proof:}

\section{$N \equiv c:$}

Since $C^{\prime}=C \wedge S=\emptyset \wedge t=g_{c}$, hence $C^{\prime} \Vdash S(C) \wedge C^{\prime}, S(A) \vdash N: t$ is a typing.

$N \equiv x:$

Since $C^{\prime}=C \wedge S=\emptyset \wedge t=A(x)$, hence $C^{\prime} \Vdash S(C) \wedge C^{\prime}, S(A) \vdash N: t$ is a typing.

$N \equiv \lambda x . M:$

Let $\left(C^{\prime}, S, t^{\prime}\right)=T Y P E(C, A ; x: \alpha, M)$. By hypothesis

$$
C^{\prime} \Vdash S(C) \wedge C^{\prime}, S(A) ; x: S(\alpha) \vdash M: t^{\prime}
$$

is a typing. Therefore, $C^{\prime}, S(A) \vdash \lambda x \cdot M: S(\alpha) \rightarrow t^{\prime}$ is a typing.

$N \equiv M_{1} M_{2}:$

Let $\left(C_{1}, S_{1}, t_{1}\right)=T Y P E\left(C, A, M_{1}\right)$ and $\left(C_{2}, S_{2}, t_{2}\right)=T Y P E\left(C_{1}, S_{1}(A), M_{2}\right)$. By hypothesis, we have:

(1) $C_{1}, S_{1}(A) \vdash M_{1}: t_{1}$ is a typing and $C_{1} \Vdash S_{1}(C)$.

(2) $C_{2}, S_{2} S_{1}(A) \vdash M_{2}: t_{2}$ is a typing and $C_{2} \Vdash S_{2}\left(C_{1}\right) \Vdash-S_{2} S_{1}(C)$.

which in turn entail the following consequences:

(1') $C^{\prime}, R^{\prime} R S_{2} S_{1}(A) \vdash M_{1}: R^{\prime} R(\alpha) \rightarrow R^{\prime} R(\beta)$ is a typing, as $R^{\prime} R(\alpha) \rightarrow R^{\prime} R(\beta)=R^{\prime} R S_{2}\left(t_{1}\right), C^{\prime}\left\|-R^{\prime} R\left(C_{2}\right)\right\|-R^{\prime} R S_{2}\left(C_{1}\right)$, and typing is closed under instance relation.

(2) $C^{\prime}, R^{\prime} R S_{2} S_{1}(A) \vdash M_{2}: R^{\prime} R\left(t_{2}\right)$ is a typing, as $C^{\prime} \Vdash R^{\prime} R\left(C_{2}\right)$ and typing is closed under instance relation.

Combining the typings in $\left(1^{\prime}\right)$ and $\left(2^{\prime}\right)$, we conclude that $C^{\prime}, R^{\prime} R S_{2} S_{1}(A) \vdash M_{1} M_{2}: R^{\prime} R(\beta)$ is a typing, as $C^{\prime} \Vdash\left\{R^{\prime} R\left(t_{2}\right) \triangleright R^{\prime} R(\alpha)\right\}$. Since $C^{\prime} \Vdash R^{\prime} R S_{2}\left(C_{1}\right)$ and $C_{1} \Vdash S_{1}(C), C^{\prime} \Vdash R^{\prime} R S_{2} S_{1}(C)$.

In the following proof for syntactic completeness, we will assume the fact:

\section{FACT:}

Let $\left(C^{\prime}, S, t\right)=T Y P E(C, A, N)$ succeeds.

If $v$ does not occur in $\left.A\right|_{F V(N)}$ and $v$ is not created by $T Y P E$ then $S(v)=v$.

This corresponds to the intuition: if the type inference algorithm TYPE does not refer to variable $v$ it does not affect $v$ at all.

\section{Theorem 7 TYPE is Complete}

If $C_{*}, A_{*} \vdash N: t_{*}$ is a typing and $\exists T$ s.t. $C_{*} \|-\left.T(C) \wedge A_{*}\right|_{F V(N)}=\left.T(A)\right|_{F V(N)}$ then $\left(C^{\prime}, S, t\right)=$ $T Y P E(C, A, N)$ succeeds and $C_{*}, A_{*} \vdash N: t_{*}$ is an instance of $C^{\prime}, S(A) \vdash N: t$.

proof:

$N \equiv c:$

Since $C^{\prime}=C \wedge S=I d \wedge t=g_{c}$, we can choose $\gamma$ to be $T$.

$N \equiv x:$

Since $C^{\prime}=C \wedge S=I d \wedge t=A(x)$, we can choose $\gamma$ to be $T$. 
$N \equiv \lambda x \cdot M:$

By the definition of typing, $\exists r_{1}, r_{2}$ such that

$$
C_{*}, A_{*} ; x: r_{1} \vdash M: r_{2} \wedge C_{*} \|\left\{r_{1} \rightarrow r_{2} \triangleright t_{*}\right\}
$$

Let $T^{\prime}=T\left[\alpha+r_{1}\right]$ such that $\left.\left(A_{*} ; x: r_{1}\right)\right|_{F V(N)}=\left.\left(T^{\prime}(A ; x: \alpha)\right)\right|_{F V(N)}$ and $C_{*} \|-T^{\prime}(C)$. By hypothesis, $\exists \gamma$ such that:

- $C_{*} \|-\gamma\left(C^{\prime}\right)$

- $\left.A_{*}\right|_{F V(\lambda x . M)}=\left.\gamma(S(A))\right|_{F V(\lambda x . M)}$, as

$\left.\left(A_{*} ; x: r_{1}\right)\right|_{F V(M)}=\left.(\gamma(S(A) ; x: S(\alpha)))\right|_{F V(M)}$

- $C_{*} \|-\left\{\gamma(t) \triangleright r_{2}\right\}$

The last condition to show is that $C_{*} \|\left\{\gamma(S(\alpha) \rightarrow t) \triangleright r_{1} \rightarrow r_{2}\right\}$. Since $C_{*} \|\left\{\gamma(t) \triangleright r_{2}\right\}$, it is enough to show $C_{*} \mathbb{H}\left\{r_{1} \triangleright \gamma(S(\alpha))\right\}$. If $x \in F V(M)$ then we are done, as $\left.\left(A_{*} ; x: r_{1}\right)\right|_{F V(M)}=(\gamma(S(A) ; x:$ $S(\alpha)))\left.\right|_{F V(M)}$. Otherwise $\alpha$ does not occur in $C^{\prime}, S$, and $t$ and we can choose $\gamma$ to be $\gamma\left[\alpha \leftarrow r_{1}\right]$ without affecting the other conditions.

$N \equiv M_{1} M_{2}:$

By the definition of typing, $\exists r_{1}, r_{2}$ such that:

- $C_{*}, A_{*} \vdash M_{1}: r_{1} \rightarrow r_{2}$

- $C_{*}, A_{*} \vdash M_{2}: r_{1}$

- $C_{*} \|-\left\{r_{2} \triangleright t_{*}\right\}$

By hypothesis and FACT $\exists \gamma_{1}$ :

(1) $\left.A_{*}\right|_{F V\left(M_{1} M_{2}\right)}=\left.\left(\gamma_{1}\left(S_{1}(A)\right)\right)\right|_{F V\left(M_{1} M_{2}\right)}$

(2) $C_{*} \| \gamma_{1}\left(C_{1}\right)$

(3) $C_{*} \|-\left\{\gamma_{1}\left(t_{1}\right) \triangleright r_{1} \rightarrow r_{2}\right\}$

Similarly, applying hypothesis to the typing corresponding to $M_{2}$ and by FACT, $\exists \gamma_{2}$ such that:

(1) $\left.A_{*}\right|_{F V\left(M_{1} M_{2}\right)}=\left.\left(\gamma_{2}\left(S_{2}\left(S_{1}(A)\right)\right)\right)\right|_{F V\left(M_{1} M_{2}\right)}$

$(2)^{\prime} \quad C_{*} \|-\gamma_{2}\left(C_{2}\right) \Vdash-\gamma_{2}\left(S_{2}\left(C_{1}\right)\right)$ (Since $C_{2} \Vdash-S_{2}\left(C_{1}\right)$ )

(3) $C^{\prime} \|-\left\{\gamma_{2}\left(t_{2}\right) \triangleright r_{1}\right\}$

By FACT, $S_{2}$ can only act on type variables in $t_{1}$ that occur in $S_{1}(A)$. By (1) and (1)', for such variables $\gamma_{2} \circ S_{2}$ must act exactly as $\gamma_{1}$ does. Therefore we can rewrite (3) to:

$(3)^{\prime \prime} \quad C_{*} \|-\left\{\gamma_{2}\left(S_{2}\left(t_{1}\right)\right) \triangleright r_{1} \rightarrow r_{2}\right\}$

Since $\gamma_{2}$ instantiates $S_{2}\left(t_{1}\right)$ to an "arrow" type, by the property of UNIFY $\exists \gamma_{3}$ such that:

(a) $\gamma_{2}=\gamma_{3} \circ R$

The following entailments are consequence of $(a),(3)^{\prime}$, and $(3)^{\prime \prime}$ :

(b) $C_{*} \|-\left\{\gamma_{3}\left(R\left(t_{2}\right) \triangleright R(\alpha)\right)\right\}$

(c) $C_{*} \|-\left\{\gamma_{3}(R(\beta)) \triangleright r_{2}\right\}$

Finally, by $(b)$ and the property of MATCH $\exists \gamma$ such that: 
(d) $\gamma_{3}=\gamma \circ R^{\prime}$

From the arguments above we conclude that:

- $\left.A_{*}\right|_{F V\left(M_{1} M_{2}\right)}=\left.\gamma R^{\prime} R S_{2} S_{1}(A)\right|_{F \vee\left(M_{1} M_{2}\right)}\left(\mathrm{By}(1)^{\prime},(a)\right.$, and $\left.(d)\right)$

- $C_{*} \|\left\{\gamma R^{\prime} R(\beta) \triangleright t_{*}\right\}$ (By $(c),(d)$, and $\left.C_{*} \|\left\{r_{2} \triangleright t_{*}\right\}\right)$

- $C_{*} \Vdash \gamma\left(C^{\prime}\right)\left(\mathrm{By}(2)^{\prime},(a),(b)\right.$, and $\left.(d)\right)$

There are two comments to our completeness result. First, the theorem is still true if we change condition $\left.A_{*}\right|_{F V(N)}=\left.T(A)\right|_{F V(N)}$ in the theorem to $\left.\left.C_{*} \mathbb{f} A_{*}\right|_{F V(N)} \triangleright T(A)\right|_{F V(N)}$. Secdondly, in the proof, instead of showing $C_{*} \|-\left.\left.A_{*}\right|_{F V(N)} \triangleright \gamma(S(A))\right|_{F V(N)}$ we show a stronger result: $\left.A_{*}\right|_{F V(N)}$ is actually equal to $\left.\gamma(S(A))\right|_{F V(N)}$ which does not fully use the flexibility of the definition for instance. This is no longer true after we minimize the principal typings computed by $T Y P E$. 